Check for updates

Cite this: Mater. Adv., 2020, 1,3387

Received 18th August 2020, Accepted 26th October 2020

DOI: $10.1039 / \mathrm{d} 0 \mathrm{ma} 00619 \mathrm{j}$

rsc.li/materials-advances

\section{A novel receptor-free polydiacetylene nanofiber biosensor for detecting $E$. coli via colorimetric changes $\dagger$}

\author{
Abhishek Bhattacharjee, ${ }^{a}$ Richard Clark, ${ }^{\mathrm{b}}$ Claudia Gentry-Weeks ${ }^{\mathrm{b}}$ and \\ Yan Vivian Li iD *acd
}

\begin{abstract}
A nanofiber-based polydiacetylene (PDA) biosensor was prepared with polyurethane (PU) to detect Escherichia coli. The main advantage of this PU-PDA nanofiber biosensor was the simplicity of the preparation method via electrospinning where no complex receptors were required to detect $E$. coli via colorimetric properties. Diacetylene monomers self-assembled during the electrospinning process that significantly enhanced the interaction between the PDA macromolecules and E. coli, resulting in high sensitivity of bacterial detection. Experiments showed that the PU-PDA nanofiber system was highly sensitive and could quickly detect $E$. coli by a colorimetric transition that is visible to the naked eye. The colorimetric property of the PU-PDA nanofiber was highly sensitive to organic solvents, suggesting potential for application in fingerprinting devices capable of distinguishing organic solvents. Additional experiments indicated that the color-changing substances (CCS) in the complex E. coli culture were not cell-associated, rather $E$. coli secretion associated. Further experiments were conducted to understand the nature of the CCS in E. coli culture and the results showed that the CCS are free extracellular polymeric substances (EPS) in E. coli culture.
\end{abstract}

\section{Introduction}

$\pi$-Conjugated polymers (CP) with unique optical and electrochemical properties have been used in several applications including solar cells, ${ }^{1}$ light-emitting diodes, ${ }^{2}$ photovoltaic cells, ${ }^{3}$ sensors, ${ }^{4}$ artificial muscles, ${ }^{5}$ and laser technology. ${ }^{6}$ Due to the delocalized $\pi$ electrons along the macromolecular backbone, CPs can transfer nonspecific interactions in the molecular structures into transducible responses, ${ }^{7}$ resulting in characteristic optical and electrical properties. Polydiacetylene (PDA) is an attractive $\mathrm{CP}$ due to its quick chromatic responses upon exposure to various external stimuli such as solvents, temperature, electricity, light, mechanical stress, chemical stress, viruses, and bacteria. ${ }^{8}$ PDAs have also been used in microorganism sensing, ${ }^{9}$ drug delivery,${ }^{10}$ supercapacitors, ${ }^{11}$ and oil-water separations. ${ }^{12}$ From our lab, Alam et al. and Yapor et al. reported that PDA nanofiber composites show rapid blue-to-red

\footnotetext{
${ }^{a}$ Department of Design and Merchandising, Colorado State University, Fort Collins, USA.E-mail: yan.li@colostate.edu

${ }^{b}$ Department of Microbiology, Immunology and Pathology, Colorado State University, Fort Collins, USA

${ }^{c}$ School of Biomedical Engineering, Colorado State University, Fort Collins, USA

${ }^{d}$ School of Advanced Materials Discovery, Colorado State University, Fort Collins, USA

$\dagger$ Electronic supplementary information (ESI) available. See DOI: 10.1039/ d0ma00619j
}

color transition responding to bacteria, heat, and $\mathrm{pH}$, suggesting their potential for use in biosensor applications, especially in developing point-of-care devices such as smart wound dressings that can be used to detect bacterial infection in wound care. ${ }^{13,14}$ Point-of-care devices are recently of great interest in hospitals, battlefields, and nursing homes owing to many advantages over traditional testing technologies, such as simplicity, affordability, and effective and efficient patient care. ${ }^{15}$

10,12-Pentacosadiynoic acid (PCDA) is a common monomer to prepare PDAs via the 1,4-addition reaction of PCDAs followed by photo-polymerization via UV or $\gamma$ irradiation. Mainly, substrate-based and solution-based PDA sensors have been reported for sensor applications due to easy fabrication, cellmimicry, stability, and enhanced sensitivity. ${ }^{8}$ Although solution-based PDA sensors provide advantages in biomedical applications especially due to a high degree of cell mimicry, they are often unstable due to the aggregation and precipitation of PDA molecules. ${ }^{16}$ The substrate-based PDA sensors overcome this drawback because they are relatively stable and sensitive in many sensing applications. ${ }^{16}$ Nevertheless, to detect pathogens such as bacteria via PDA based sensors, both substrate and solution-based PDAs were reported that can sense either the bacterial cell or the materials bacteria secrete into the environment during the growth phase. Complex receptors such as specific bacterial antibodies were incorporated into the PDA sensing system that reacted with the bacterial cell and 
thus detected the presence of bacteria. Park et al. reported a glass-supported PDA based sensing system where different bacterial antibodies were used to detect bacteria via colorimetric transition. ${ }^{17}$ An indirect approach is to detect the bacterial secretion where amine functionalization of the PDA monomer and incorporation of complex receptors were performed to facilitate the reaction between the aminefunctionalized PDA and bacterial secretion materials. For instance, Villalobos et al. prepared a PDA based sensing system via amine functionalization of the diacetylene monomer that could detect bacterially secreted materials (supernatant fluid from a bacterial culture) by colorimetric transition after $4 \mathrm{~h}$ of incubation. ${ }^{18}$ In addition to the receptors, lipids such as DMPC (dimyristoyl-sn-glycero-3-phosphocholine) are often used in the PDA based sensors that mimic the host cell surface and enhance the sensitivity of the system. Kim et al. reported the effect of lipid insertion in the PDA system where an increased amount of lipids exhibited higher chromatic response when bacteria were introduced into the system. ${ }^{19}$ dos Santos Pires et al. reported a similar trend, where no significant color change was found when pure diacetylene monomer PCDA (10,12-pentacosadiynoic acid) was used, but a significant color change was observed when TDER ( $N$-[(2-tetradecanamide)ethyl]-ribonamide) was incorporated into the PCDA. ${ }^{20}$ However, the preparation of these PDA sensing systems using complex functionalization with receptors and lipids is complicated and often demands sophisticated machinery and personnel, which is a shortcoming of the existing PDA based biosensors.

This paper reports a simple, receptor-free fabrication strategy of preparing PDA biosensors capable of detecting $E$. coli via a blue-to-red colorimetric transition. PDA nanofiber composites were prepared using an electrospinning method and the stretching on the nanofibers due to electrostatic forces enhanced the self-assembly of the PCDA monomer clusters, resulting in a flexible, powerless, receptor-free PDA nanofiber biosensor potentially for many medical applications. The color change of the PDA nanofibers in response to the $E$. coli grown in LB media was visible to the naked eye with a response time of 0.5-3 min that was significantly short, suggesting a sensitive detection. The critical bacterial concentration (CBC) that was defined as a concentration initiating a colorimetric transition in the PDA nanofibers was found to be approximately $9 \times$ $10^{8} \mathrm{CFU} \mathrm{mL} \mathrm{m}^{-1}$, suggesting an effective and sufficient detection limit for practical uses in sensors. When the nanofibers were tested with two distinct components of $E$. coli culture, it was found that the colorimetric response $(\mathrm{CR} \%)$ in the nanofibers tested with supernatant fluid was significant (CR $=10 \%)$. The colorimetric response of the nanofibers was further increased to $28 \%$ by the autoclaved supernatant fluid. In comparison, no color change was found in testing with $E$. coli cell pellets. The results suggested that the color-changing substances (CCS) in E. coli culture were not cell-associated rather secretion associated, which was in good agreement with the receptor-free fabrication strategy of preparing PDAs. When a minimum nutrition medium (M9 minimal medium) was used to grow E. coli, no color change was found in the PDA nanofiber.
In addition, the PDA nanofibers demonstrated colorimetric transition behaviors in response to twelve commonly used organic solvents. The percentage of colorimetric response was dependent on the nature of the solvent. Further analysis confirmed that the CCS were the extracellular polymeric substances (EPS) that E. coli produced in the process of biofilm formation. The results may expand the application of current PDA biosensors in detecting EPS that is generally the primary component of bacterial biofilms.

\section{Experimental section}

\section{Materials}

10,12-Pentacosadiynoic acid (PCDA, 98\%, GFS Organics, Columbus, $\mathrm{OH}$ ) was used as a monomer for preparing PDA. Thermoplastic polyurethane (PU) was purchased from The Lubrizol Corporation (Wickliffe, $\mathrm{OH}$ ) to be used as a matrix polymer. Tetrahydrofuran (THF, 99\%) and $N, N$-dimethylformamide (DMF, 99.8\%, extra dry, AcroSeal) were purchased from Fisher Scientific (Waltham, MA) for preparing the solvent system to synthesize PDA nanofibers.

E. coli ATCC25922 $\left(1.0 \times 10^{4} \mathrm{CFU}\right.$ per pellet $)$ was purchased from ATCC (Manassas, VA). A lysogeny Broth (LB) and M9 minimal media were used for the growth of $E$. coli. For the size-fractionation test, Amicon ${ }^{\circledR}$ Ultra-4 Centrifugal Filter Units having molecular weight cutoffs of $3 \mathrm{k}, 30 \mathrm{k}$, and $100 \mathrm{k} \mathrm{Da}$ were purchased from Millipore Sigma (Burlington, MA). The prepared lipopolysaccharide (LPS) from E. coli was purchased from Millipore Sigma (Burlington, MA). Organic solvents that were used to study solvatochromism included hexanes, xylenes, isopropyl alcohol, 1-butanol, tetrahydrofuran (THF), methanol, ethanol (200 proof), glacial acetic acid, $N, N$-dimethylformamide (DMF), chloroform $\left(\mathrm{CHCl}_{3}\right)$, acetone, and dimethyl sulfoxide (DMSO). Distilled water was used as a control. All the chemicals and solvents were used without further purification.

\section{Methods}

PU-PDA nanofiber biosensors were prepared via electrospinning and then tested with $E$. coli culture components as well as with organic solvents. Fig. 1 shows a schematic of the experimental design.

\section{PDA preparation}

PDA powders were prepared from 10,12-pentacosadiynoic acid (PCDA) monomers. $6.4 \mathrm{~g}$ of PCDA was dissolved in $35 \mathrm{~mL}$ chloroform and stirred to make a homogenous solution. The solution was then filtered to remove any contaminants. The solvent was removed from the filtered solution by continuous stirring under the hood until dry powders were isolated. Millipore water was added to make a $1.29 \% \mathrm{w} / \mathrm{v}$ suspension and the suspension was sonicated for $30 \mathrm{~min}$. After the sonication, the suspension was allowed to cool down to room temperature and stored at $4{ }^{\circ} \mathrm{C}$ for $24 \mathrm{~h}$. Then, the suspension was transferred to a glass beaker with a magnetic stir bar and irradiated with UV light at $254 \mathrm{~nm}$ for $10 \mathrm{~min}$. The color of the suspension 


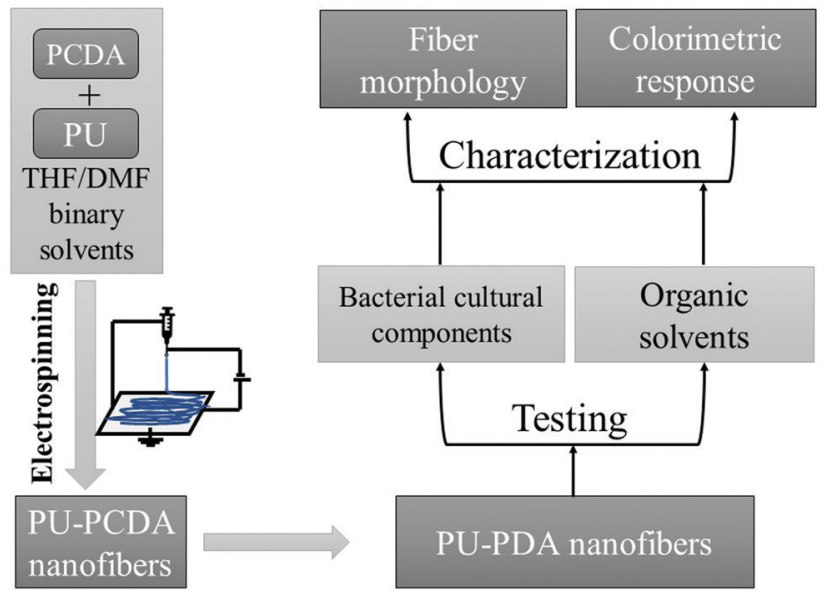

Fig. 1 Schematic of the experimental design for the study.

changed to dark blue, resulting in PDA. $7 \mathrm{~mL}$ of the dark blue suspension was taken into a centrifuge tube and centrifuged for $10 \mathrm{~min}$ at $6000 \mathrm{rpm}$. The dark blue precipitation at the bottom of the tube was taken out and kept in an open glass vial to allow the solid to completely dry, resulting in blue PDA powders.

Electrospinning of PU-PDA nanofibers. Electrospinning solutions were prepared using a method adopted from Yapor $e t$ al. ${ }^{14}$ The preparation process of PU-PDA nanofibers is provided in Section S1 of the ESI. $\dagger$ All the blue PU-PDA nanofiber mats prepared by electrospinning were stored in the dark to avoid any unwanted continuous photo-polymerization for future tests.

Fiber morphology characterization. The morphology of PU-PDA nanofibers was analyzed before and after the tests with E. coli culture components using a Scanning Electron Microscope (SEM) (JEOL JSM-6500F Field Emission Scanning Electron Microscope). PU-PDA nanofiber mats were kept at room temperature in the dark for $24 \mathrm{~h}$ to evaporate any residual solvent or moisture. Before imaging, they were sputter-coated with gold $(\mathrm{Au})$ for $33 \mathrm{~s}$ using a Denton Vacuum-DESK 2 Sputter Coater to improve conductivity. The average diameters of the fibers were calculated from SEM images using ImageJ software.

Attenuated total reflectance-Fourier transform infrared (ATR-FTIR) spectroscopy. A Nicolet 6700 FTIR spectrometer (Thermo Electron Corporation, Madison, WI, USA) was used to collect Attenuated Total Reflectance-Fourier Transform Infrared (ATR-FTIR) spectra of the obtained PDA powders, the PU-PDA nanofibers before (blue) and after (red) bacterial testing.

X-ray diffraction (XRD). X-ray diffraction (XRD) spectra of the obtained PDA powders and the PU-PDA nanofibers (blue and red) were collected using a Bruker D8 Discover DaVinci X-ray diffractometer (for the PDA powder) and a Bruker D8 Discover Series I X-ray diffractometer (for PU-PDA nanofibers).

Culture medium preparation. LB and M9 minimal media were prepared following general microbiology procedures. Detailed preparation methods are provided in Section S2 of the ESI. $\dagger$

Preparation of $\boldsymbol{E}$. coli culture components. A premade E. coli ATCC25922 pellet was hydrated in the hydration liquid following the manufacturer's procedures. After hydration, the mixture was vortexed and added to $250 \mathrm{~mL} \mathrm{LB}$ medium and incubated at $37{ }^{\circ} \mathrm{C}$ for $24 \mathrm{~h}$. After that, an inoculation loop was used to transfer the bacteria to an LB streak plate and placed in an incubator to grow overnight at $37^{\circ} \mathrm{C}$. Next, a single colony was taken from the streak plate and placed in $500 \mathrm{~mL} \mathrm{LB}$ medium and incubated at $37{ }^{\circ} \mathrm{C}$ using a shaker incubator for $24 \mathrm{~h}$. The $E$. coli culture then was centrifuged at $11984 \times g$ for $10 \mathrm{~min}$ at $27{ }^{\circ} \mathrm{C}$. The supernatant fluid was collected, filtered $(0.2 \mu \mathrm{m}$ syringe polyethersulfone filters), and divided evenly into two flasks. One half of the supernatant fluid was autoclaved at $121.11^{\circ} \mathrm{C}$ at $23 \mathrm{psi}$ for $20 \mathrm{~min}$ and the other half was kept in the incubator at room temperature. On the other hand, the obtained bacterial cell pellet after centrifugation was washed twice using a $0.2 \%$ sterile saline solution and centrifuged again at $11984 \times g$ for $10 \mathrm{~min}$ at $27{ }^{\circ} \mathrm{C}$. The cell pellets were then dispersed in a $0.9 \%$ saline solution and separated into two flasks. One half of the cell pellet solution was autoclaved at $121.11{ }^{\circ} \mathrm{C}$ at $23 \mathrm{psi}$ for $20 \mathrm{~min}$ and the other half was kept at room temperature. Besides, two supplementary batches of supernatant fluid were prepared following the same procedures described above. One batch of supernatant fluid (including both autoclaved and non-autoclaved) was used to treat PU-PDA nanofibers at $24 \mathrm{~h}\left(t_{\mathrm{s}}=24 \mathrm{~h}\right)$ storage time at room temperature. The other batch was kept for $36 \mathrm{~h}\left(t_{\mathrm{s}}=36 \mathrm{~h}\right)$ storage time. $E$. coli culture in M9 minimal media was prepared and divided into four component solutions following the procedure described for LB. To summarize, the E. coli culture components included (1) autoclaved supernatant fluid, (2) non-autoclaved supernatant fluid, (3) autoclaved bacterial cell pellets, and (4) nonautoclaved bacterial cell pellets from both LB and M9 minimal media. LB and M9 minimal medium solutions were used as controls.

Size fractionation of supernatant fluid. Supernatant and autoclaved supernatant fluids were prepared using the methods described above from LB culture media. $4 \mathrm{~mL}$ of non-autoclaved supernatant fluid was added to each Amicon ${ }^{\circledR}$ Ultra-4 Centrifugal Filter Unit having molecular weight cutoffs of $3 \mathrm{k}, 30 \mathrm{k}$, and $100 \mathrm{k}$ Da and centrifuged at $4000 \times g$ for $15 \mathrm{~min}$ according to the manufacturer's procedures. The filtrate fluid was collected and used for testing PU-PDA nanofibers. The same procedures were followed for autoclaved supernatant fluid. Six fluid samples containing molecules with a molecular weight less than $3 \mathrm{k}$, $30 \mathrm{k}$, and $100 \mathrm{k}$ Da were collected to test on PU-PDA nanofibers. The fluid that did not pass through the 100k filter device having a molecular weight greater than 100k Da was also collected from the supernatant and autoclaved supernatant fluid to test on PU-PDA nanofibers.

Free EPS extraction. Free EPS was extracted from the supernatant fluid using a method adopted from Eboigbodin and Biggs. ${ }^{21}$ Supernatant fluid from LB culture media was centrifuged for $30 \mathrm{~min}$ at $11984 \times g$ and $4{ }^{\circ} \mathrm{C}$, decanted, and ethanol was mixed with the decanted solution. After the container was inverted 24 times and stored at $-20{ }^{\circ} \mathrm{C}$ for $18 \mathrm{~h}$, the precipitate was pelleted by centrifugation at $11,984 \times g$ for $15 \mathrm{~min}$ at $4{ }^{\circ} \mathrm{C}$, resulting in free EPS. The free EPS pellet was suspended in $70 \%$ ethanol and the suspension was washed with distilled water. 
Residual ethanol was evaporated from free EPS suspension by keeping it at room temperature for 48 h. $5 \mathrm{~mL}$ distilled water was added to the suspension and vortexed. The resultant solution was used to test on PU-PDA nanofibers.

Colorimetric transition behaviors of PU-PDA nanofibers. The nanofiber samples were immersed in E. coli culture component solutions including (1) autoclaved supernatant fluid, (2) non-autoclaved supernatant fluid, (3) bacterial cell pellets, and (4) non-autoclaved bacterial cell pellets. The immersion time was varied in a range of 1, 2, 3, 30, 60, 90, 120, 150, $180 \mathrm{~min}$, and $24 \mathrm{~h}$. Colorimetric properties of the PU-PDA nanofibers upon testing with bacterial culture components were evaluated first visually and then using a spectrophotometer (ColorQuest, Hunter Lab). A PU-PDA nanofiber mat ( 2 inch $\times 2$ inch) was placed inside a sterile Petri dish, photographed and a spectrophotometer measurement was taken before testing. Then each culture component solution $(25 \mathrm{~mL})$ was poured slowly on the nanofiber mat until the mat was completely immersed. After $1 \mathrm{~min}$, the nanofiber mat was taken out and placed in another sterile Petri dish for photograph recording and spectrophotometry measurement $(t=1 \mathrm{~min})$. After that, the nanofiber mat was immersed back into the same culture component solution for an additional $1 \mathrm{~min}$, and then photographed and measured with a spectrophotometer $(t=2 \mathrm{~min})$. This process was repeated, and photographs and spectrophotometer measurements were taken for each of the measurement times $(t=1,2,3$, $30,60,90,120,150,180 \mathrm{~min}$, and $24 \mathrm{~h}$ ) and treatment with each culture component. Fiber mats were also immersed in LB and M9 minimal media (having no $E$. coli cells or materials produced by $E$. coli) for control testing. Besides, the free EPS solution was used to test with PU-PDA nanofibers as well. All the tests were repeated three times and the average values of spectrophotometer measurements were taken for further calculations. The colorimetric response percentage (CR\%) was calculated using the method described by Yapor et al. ${ }^{14}$

Lipopolysaccharide (LPS) solution preparation. Lipopolysaccharide (LPS) powder was suspended in $1 \mathrm{~mL}$ of distilled water and $1 \mathrm{~mL}$ of LB broth at a 1:10 ratio for each solution. Then, the solutions were used to test the PU-PDA nanofibers in a tissue culture well. The nanofiber mats were immersed in the LPS solutions for $72 \mathrm{~h}$.

Critical bacterial concentration (CBC). To calculate the concentration of $E$. coli required for initiating a colorimetric transition in PU-PDA nanofibers, the LB medium was used to culture E. coli. From the beginning of the culture $(0 \mathrm{~h})$, optical density (OD) reading using a Thermo Scientific ${ }^{\mathrm{TM}}$ GENESYS $^{\mathrm{TM}}$ 20 Visible Spectrophotometer was collected at $600 \mathrm{~nm}$ with a $1 \mathrm{~h}$ interval. When a significant change in OD600 reading was observed, the PU-PDA nanofiber was tested with the E. coli culture solution instantly and reflectance data were collected by the method described earlier. E. coli CFU/mL and PU-PDA nanofibers' CR\% were calculated from OD600 readings and reflectance measurements respectively.

Colorimetric transition behaviors of PU-PDA nanofibers in common organic solvents. A PU-PDA nanofiber mat $(2$ inch $\times$ 2 inch) was placed inside a Petri dish, photographed and spectrophotometer measurement was taken before testing with an organic solvent. Then one organic solvent $(25 \mathrm{~mL})$ was poured slowly on the nanofiber mat until it was completely immersed. The nanofiber mat was immersed in the solvent for at least $30 \mathrm{~min}$. Then the nanofiber mat was taken out of the Petri dish and placed in a new sterile Petri dish for photography and spectrophotometer measurement. The experiment was repeated three times for each of the 12 solvents studied. Then CR\% was calculated from the spectrophotometer data.

\section{Results and discussion}

\section{Electrospinning and PU-PDA nanofiber morphology}

In the electrospinning of nanofibers, large electrostatic forces due to high voltage enable molecular orientation in a nanofiber when the nanofiber is drawn and produced. The large electrostatic forces have been reported to be highly efficient in enhancing fiber orientation and hence improving the mechanical performance of the fibers. ${ }^{22-24}$ In the electrospinning of PCDA and PU mixture solution, PCDA monomers were randomly distributed in the PU solution. Fig. 2a shows X-ray diffraction (XRD) spectra of blue electrospun PU-PDA nanofibers and non-electrospun PDA powders. Significant differences were found between the XRD crystallography of electrospun PDA nanofibers and non-electrospun PDA powders. A broad peak at a $2 \theta$ value of $12.05^{\circ}$ was observed in the XRD spectra of the PDA powder, indicating a large amorphous region, ${ }^{25}$ which was absent in the spectra of PU-PDA nanofiber, confirming improved crystallinity in the fiber compared to the powder (Fig. 2a). Sharp and strong peaks at $2 \theta$ values of $5.62^{\circ}, 9.39^{\circ}, 38.48^{\circ}$, and $44.71^{\circ}$ found in the XRD spectra of the PDA nanofiber suggesting significant enhancement of crystallinity in the PDA nanofiber in comparison with PDA powders. It has been previously reported that large external forces could overcome the molecular attraction between PCDA monomers and PU molecules, thus promoting molecular alignment in PCDA monomers, resulting in PCDA selfassembly structures. ${ }^{26}$ During electrospinning, large electrostatic forces were able to significantly align the PCDA monomers and therefore enhance the self-assembly structures of PCDA. An illustration scheme shown in Fig. 2b demonstrates the molecular alignment and self-assembly of PCDA, as well as the PDA molecular vesicles after the UV polymerization.

Fig. 3 shows the SEM images of the PU nanofiber, PU-PDA nanofiber, and the nanofibers interacting with different components from $E$. coli culture. The PU nanofibers had a diameter ranging from 200-300 nm. The addition of PDA in the PU nanofibers increased the resultant fiber diameter ranging from 200-2000 nm. A flake-like structure on the PU-PDA fiber surface (Fig. 3b-f) was found compared to the PU nanofibers. ${ }^{27}$ No significant change in fiber morphology (Fig. 3c-f) was found in the nanofibers after the colorimetric detection of $E$. coli occurred in later testing.

\section{Sensitivity of detecting $E$. coli-critical bacterial concentration}

Critical bacterial concentration (CBC) is defined as the concentration of bacteria at which colorimetric transition in PDA 


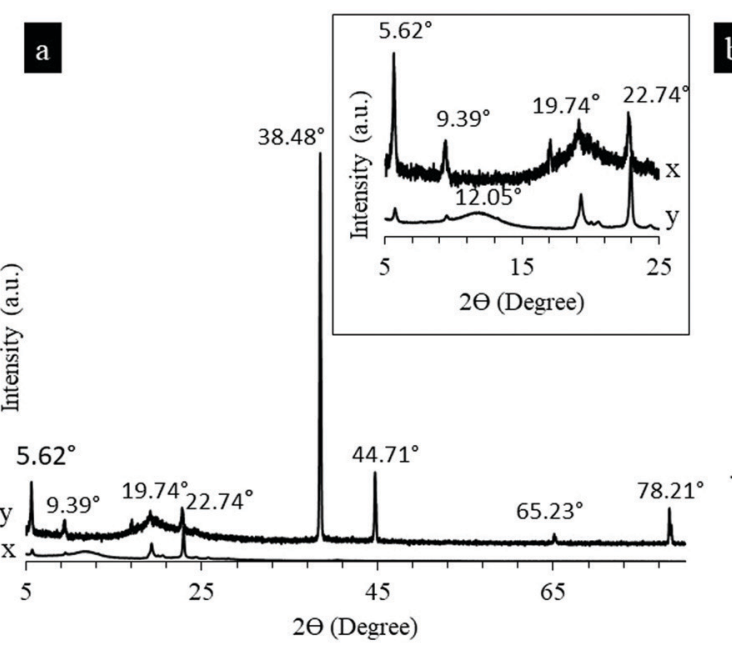

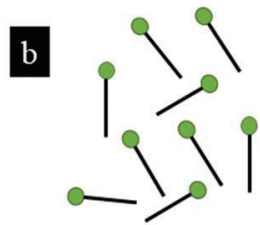

Randomly distributed PCDA monomers in electrospinning solution

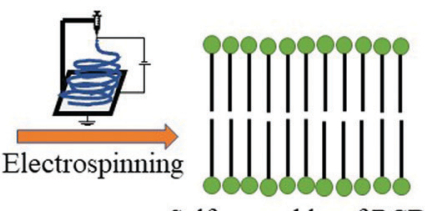

Self-assembly of PCDA monomers during electrospinning

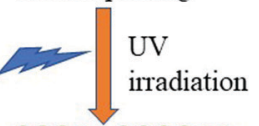

○

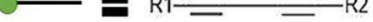

PCDA monomer polymerized to PDA and color changed to blue

Fig. 2 (a) XRD spectra of the non-electrospun PDA powder $(x)$ and electrospun PU-PDA nanofiber ( $y$ ). Zoomed-in XRD spectra are shown in the inset having $2 \theta$ values from $5^{\circ}$ to $25^{\circ}$. (b) Schematic of self-assembly and polymerization of PCDA into PDA.

occurs. The CBC determines the sensitivity of using the PU-PDA nanofiber for the identification of bacterial presence in point-of-care devices such as smart wound dressings. The colony-forming unit per volume $\left(\mathrm{CFU} \mathrm{mL} \mathrm{m}^{-1}\right)$ of E. coli is a common indicator of bacterial concentration. Fig. 4a shows a plot of colorimetric response (CR\%) of the PU-PDA nanofiber as a function of $E$. coli concentration in $\mathrm{CFU} \mathrm{mL}^{-1}$. The color change in PU-PDA nanofibers began to occur immediately when exposed to the $E$. coli concentration that was approximately $9 \times 10^{8} \mathrm{CFU} \mathrm{mL}^{-1}$. The nanofiber mat started changing color in some spots immediately at the CBC and then completely changed in red after a couple of minutes. The response time was nearly consistent with an increase in bacterial concentration. The bacterial concentration in wounds causing infection
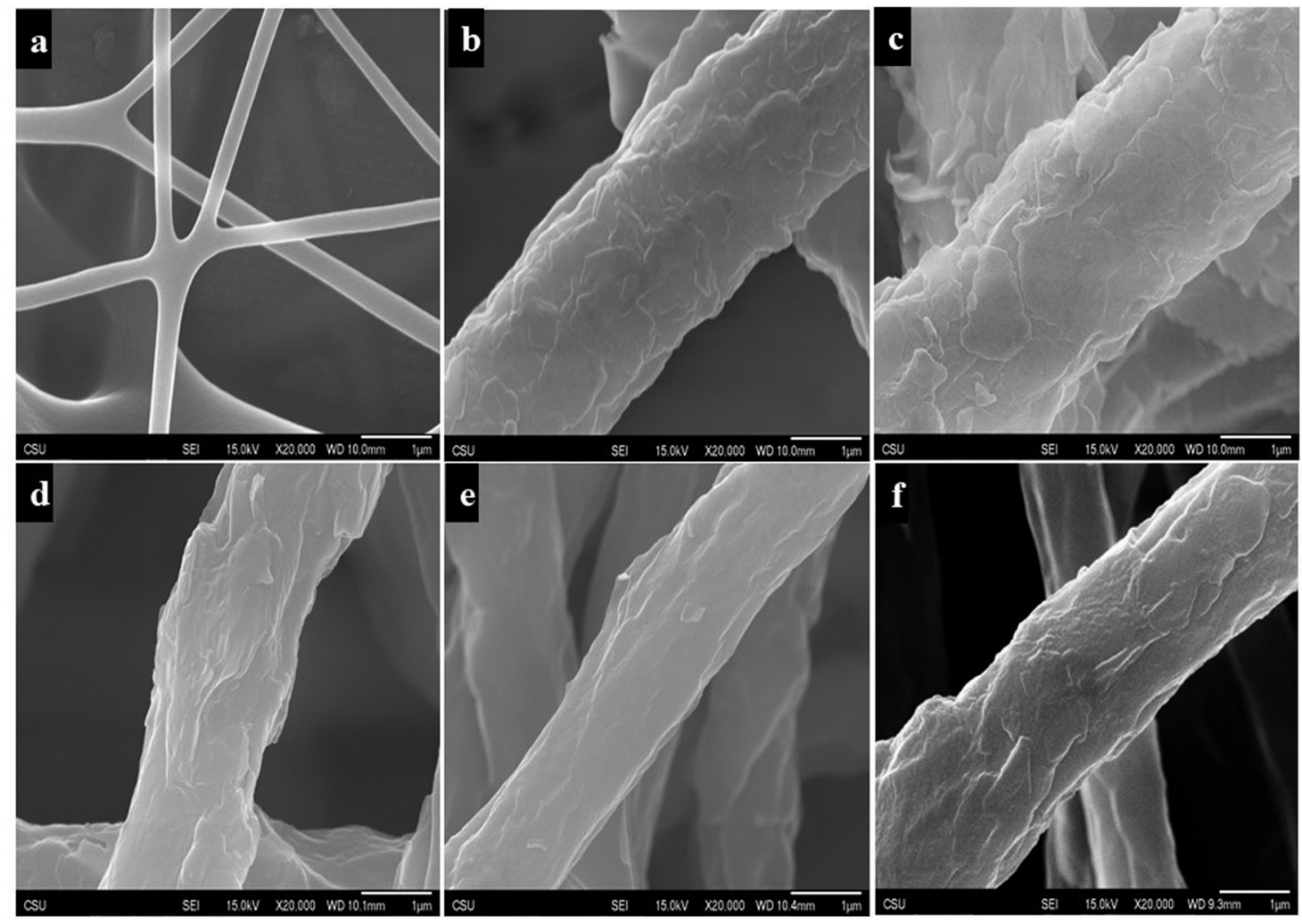

Fig. 3 SEM image of (a) the pristine PU nanofiber and (b) PU-PDA nanofibers before testing with bacterial components, PU-PDA nanofibers after testing with (c) autoclaved supernatant fluid, (d) non-autoclaved supernatant fluid, (e) autoclaved cell pellet, and (f) non-autoclaved cell pellet. 


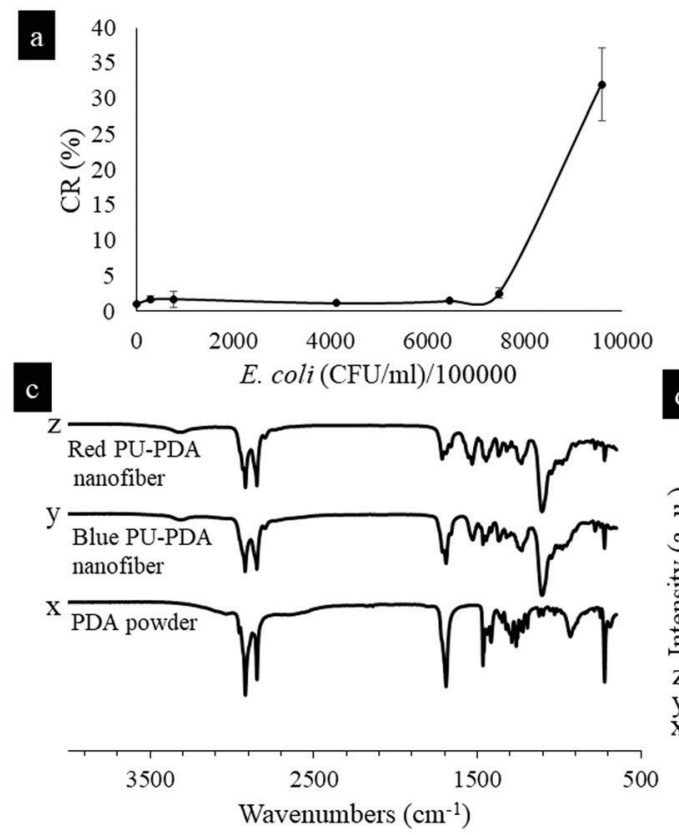

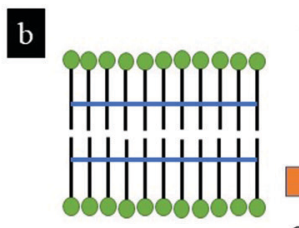

Before E. coli exposure

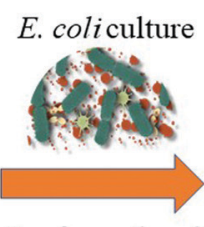

Conformational change in PDA

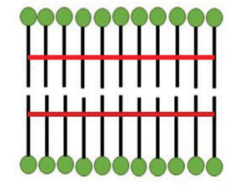

After E. coli exposure
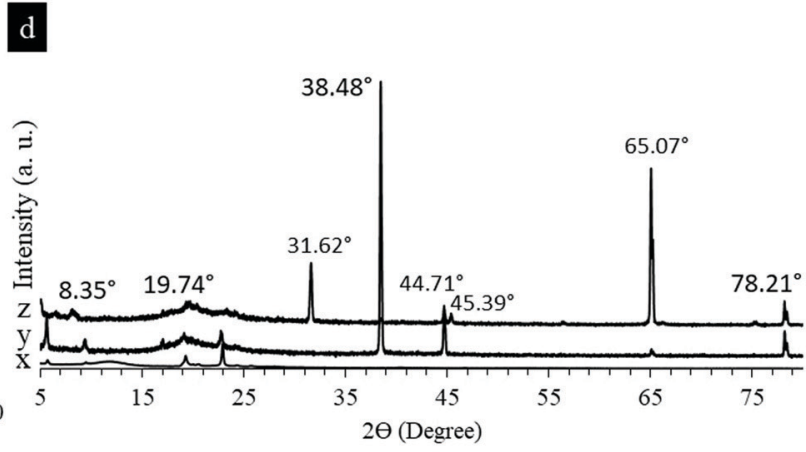

Fig. 4 (a) Colorimetric response (CR) of PU-PDA nanofibers with E. coli concentration measured in (CFU $\left.\mathrm{mL}^{-1}\right) / 10^{5}$; (b) schematic of conformational change in a PDA vesicle and the blue-to-red color transition in the PDA nanofibers; and (c) ATR-FTIR spectra of the PDA powder and PDA nanofibers. The signals correspond to the PDA powder ( $x$ ), blue PU-PDA nanofiber ( $y$ ), and red PU-PDA nanofiber ( $z$ ); and (d) XRD spectra of the non-electrospun PDA powder $(x)$, electrospun blue PU-PDA nanofiber $(y)$, and electrospun red PU-PDA nanofiber $(z)$.

generally lies in the range of $10^{5}$ and $10^{9} \mathrm{CFU} \mathrm{mL}{ }^{-1} \cdot{ }^{28,29}$ If the detection limit were too low, the detection response in practical applications would occur even at a bacterial level normally found in healthy skins, resulting in a false signal of sensing. Therefore, the PU-PDA nanofiber composites demonstrated sufficient sensitivity for practical infection detection in wound care. The high sensitivity of the PDA nanofibers most likely contributed to the highly ordered and self-assembled PDA vesicle nanostructures that were sensitive to external stimuli, resulting in a conformational change in the PDA vesicles and eventually a colorimetric transition from blue-to-red in the PDA nanofibers. Fig. 4b shows an illustration scheme of conformational change in a PDA vesicle and the blue-to-red color transition in the PDA nanofibers.

Previous reports on PDA suggested a conformational change in the $\pi$ conjugated electron backbone structure of PDA when the color changes from blue to red. ${ }^{13,14}$ The conformational change in PDA nanofibers was confirmed by FTIR spectra shown in Fig. 4c. A shift of characteristic peaks on the FTIR spectra between the blue nanofibers and the red nanofibers was found in carbonyl stretch from 1691 to $1715 \mathrm{~cm}^{-1}$. The carbonyl bond strength increased and the bond length shortened in the red nanofibers, suggesting a decrease in hydrogen bond strength and resulting in a disruption in the hydrogen bond along the side chain of PDAs. The disruption in the hydrogen bond initiated a conformational change in the $\pi$ electron cloud of PDA and resulted in a color change in the nanofiber composite. In addition, Fig. 4c shows the FTIR spectra of non-electrospun PDA powders. All of the characteristic peaks were present in both PDA nanofibers and powders, suggesting no differences in the chemical structures of PDA in nanofibers and powders. Fig. 4d shows XRD spectra of the PUPDA nanofibers after color change. The intense peak at $38.48^{\circ}$ found in the blue nanofibers was reduced drastically in the red nanofibers. New peaks at $8.35^{\circ}, 31.62^{\circ}, 45.39^{\circ}$, and $65.07^{\circ}$ were found in the red nanofibers in a comparison with the blue nanofibers. The XRD results suggested changes in crystallography during color shift from blue-to-red, which is most likely the result of a conformational change in the electron cloud of PDA molecules in the nanofibers.

\section{Colorimetric response of nanofibers in organic solvents}

Solvatochromism is a property that indicates a color change in molecules or materials in organic solvents. PDAs exhibit solvatochromism where the molecules turn blue-to-red when different organic solvents are introduced. Several PDA based systems have been reported in the literature to identify organic solvents ${ }^{30}$ and volatile organic compounds. ${ }^{31}$ To assess the sensitivity of the PU-PDA nanofiber biosensor, the nanofibers were immersed in 12 common organic solvents and the colorimetric responses (CR\%) were calculated. Fig. 5 shows the CR\% generated by each organic solvent as a function of the solvent polarity index. 10 out of 12 solvents were able to cause a color change in the nanofibers instantly. Hexane and methanol could not generate a significant colorimetric response. With an increase of the polarity index from 0 (hexane) to 7.2 (DMSO), the $\mathrm{CR} \%$ of the nanofibers increased exponentially by almost $80 \%$, resulting in the highest CR\% (82\%) in DMSO. As different solvents generated a different $\mathrm{CR} \%$, the PU-PDA nanofiber biosensor offers the potential to differentiate and detect specific organic solvents. However, it was also found from the tests that the fiber mats began to disrupt with an increase in the 


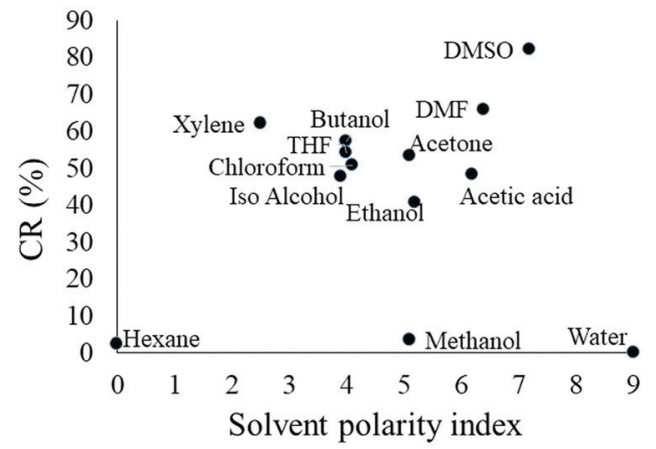

Fig. 5 CR\% of PU-PDA nanofibers with a solvent polarity index.

solvent polarity index. The solvents with a polarity index higher than 4 disassembled the fibers, resulting in serious damages at the edges of the fiber mats. The fiber mats were nearly completely dissolved in THF, acetic acid, and DMF after $30 \mathrm{~min}$ of exposure. The exception of methanol to change the color while having a polarity index the same as acetone indicates that the solvent polarity may not be the sole responsible factor for a color change in PU-PDA nanofibers. The different levels of colorimetric responses to different solvents could be very helpful in developing sensors for solvent identification.

\section{Colorimetric response of the nanofiber for $E$. coli culture components}

E. coli strain of ATCC25922 was cultured in a highly nutritious LB medium and then the $E$. coli cells were separated as pellets by centrifugation. The supernatant fluid after the centrifugation process was collected which contained all the secretion materials $E$. coli produced during the growth phase. A significant colorimetric transition from blue to red was found when the nanofiber was treated with supernatant fluid. The color change began within $30 \mathrm{~s}$ at the edges of the nanofiber mats and progressed towards the center of the mats. The response time within $30 \mathrm{~s}$ was significantly short in comparison with those reported previously, suggesting a sensitive detection offered by the PU-PDA nanofibers. ${ }^{18,32,33}$ After $3 \mathrm{~min}$, the red covered most of the nanofiber mat decorated with some blue patches. The blue patches were due to the inability of the supernatant fluid to penetrate the nanofiber mat. PU-PDA is a superhydrophobic material ${ }^{12}$ and hence the wettability of the nanofiber mat was poor. The blue patches eventually became red after $24 \mathrm{~h}$. On the other hand, no color change was observed when the nanofibers were tested with $E$. coli cell pellets or LB culture medium solution (control). The results are in good agreement with the receptor-free fabrication method of PDA nanofibers. The color change by supernatant fluid occurred most likely because the color changing substances (CCS) present in the $E$. coli secretion reacted with the highly ordered PDA vesicles in the nanofiber and triggered a molecular configuration change in the PDA vesicles, resulting in a visible colorimetric response in the PDA nanofibers. Interestingly, the observation of color change in supernatant fluid but not in cell pellets was previously reported in the PDA that was
Table 1 Photographs of PU-PDA nanofibers before and after testing with E. coli culture components and free EPS. $S=$ non-autoclaved supernatant fluid, $A S=$ autoclaved supernatant fluid, $P=$ non-autoclaved bacterial cell pellet, $\mathrm{AP}=$ autoclaved bacterial cell pellet, $\mathrm{LB}=\mathrm{LB}$ medium solution (control)

\begin{tabular}{|c|c|c|c|c|c|}
\hline E. coli component & Control & AS & $\mathrm{S}$ & AP & \\
\hline Before exposure & & & & & \\
\hline After exposure & & & & & \\
\hline
\end{tabular}

purposefully functionalized and attached with a lipid receptor to detect E. coli, Shigella sonnei, and Salmonella typhimurium. ${ }^{18}$ In comparison, the current method of electrospinning to fabricate PDA biosensors was in favor because it was a simple and cost-effective strategy in developing receptor-free PDA biosensors. Table 1 shows images of PU-PDA nanofiber mats before and after testing with $E$. coli cell pellets, supernatant fluid, and LB media (control).

Autoclaving is a sterilization process where hightemperature steam is used with high pressure to denature essential structural and enzymatic proteins, DNA, and RNA, resulting in the death of the bacteria. Proteins and nucleic acids in the bacterial supernatant fluid are denatured by the extreme heat and pressure during the autoclaving process. ${ }^{34}$ It was found that the PDA nanofiber tested with autoclaved supernatant fluid changed its color significantly faster than that tested with non-autoclaved supernatant fluid, suggesting that structural changes of CCS in the E. coli supernatant fluid occurred during autoclaving and accelerated the colorimetric response in the PDA molecules. The autoclaving experiment also indicated that the CCS may not be a protein, DNA, or RNA since they typically denature during high heat and pressure. Additionally, the colorimetric response of the PDA nanofibers was tested with lipopolysaccharide (LPS). LPS is a surface molecule that is commonly found in Gram-negative bacteria such as $E$. coli, especially in supernatant fluid, and maintains the structural integrity of the bacterial outer membrane. ${ }^{35}$ LPS was prepared separately with distilled water and LB broth to test with PU-PDA nanofibers and no color change was observed.

To quantify the colorimetric response of the PU-PDA nanofibers, reflectance data were taken for each of the nanofiber mats before and after exposure to each $E$. coli culture component at certain time intervals $(t)$. Two representative reflectance spectra of the PU-PDA nanofibers are shown in Fig. 6a and b. The spectra of the nanofibers tested in LB media showed no significant changes from $t=0$ to $t=180$ min during the test as shown in Fig. 6a, which agrees with the lack of color change in the photographs shown in Table 1 . In the case of autoclaved supernatant fluid, a peak at $540 \mathrm{~nm}$ (red) developed progressively and significantly on the spectra from $t=0$ to $t=180 \mathrm{~min}$ during the test as shown in Fig. 6b, suggesting a colorimetric transition from blue to red in the nanofiber mat as shown in 

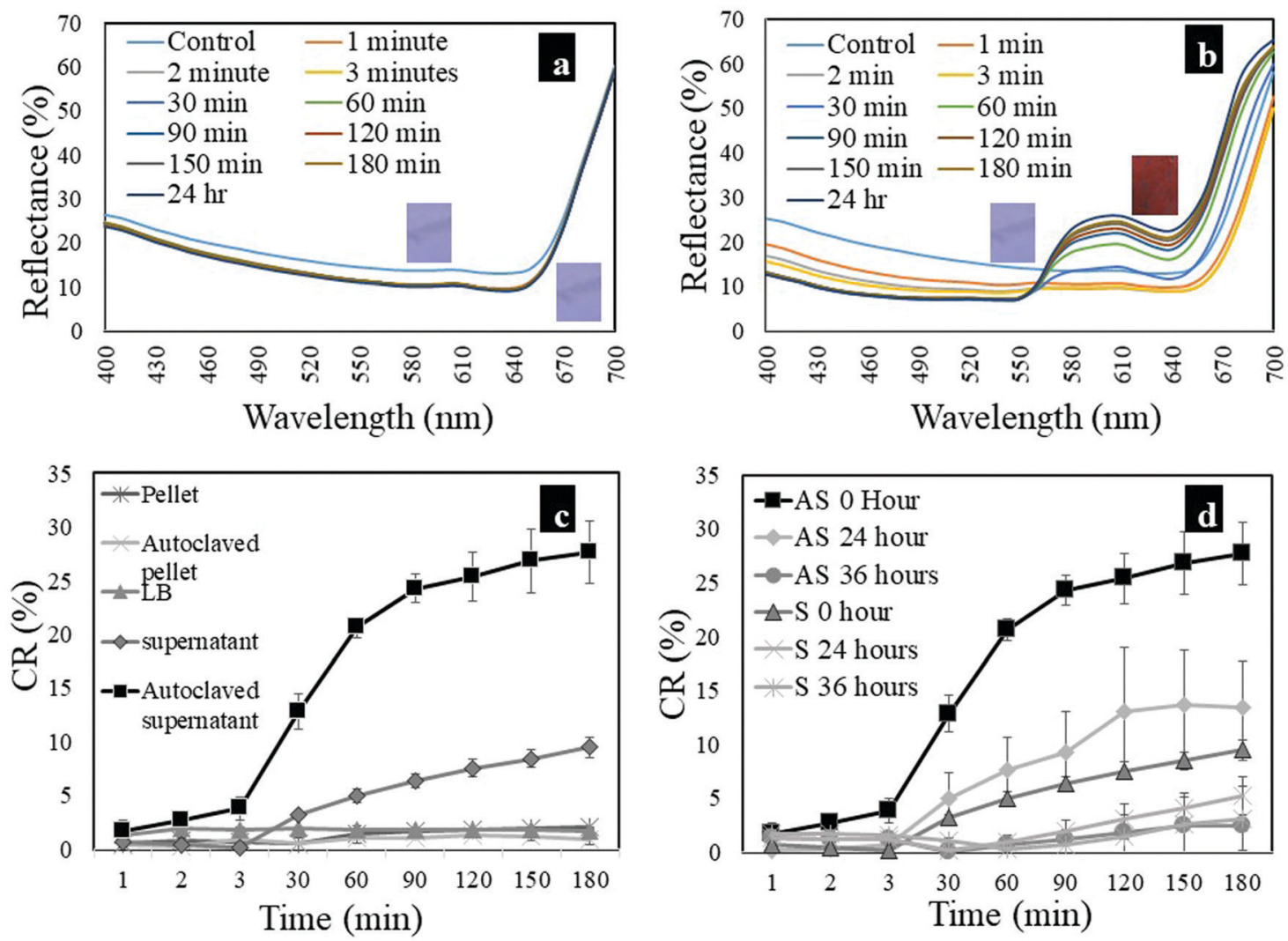

Fig. 6 Representative reflectance spectra of PU-PDA nanofibers treated with (a) LB medium (control) and (b) autoclaved supernatant fluid; (c) CR\% of PU-PDA nanofibers treated with bacterial components and (d) CR\% of PU-PDA nanofibers treated with supernatant fluid following storage for $0 \mathrm{~h}, 24 \mathrm{~h}$ and $36 \mathrm{~h}$ (AS = autoclaved supernatant fluid; $\mathrm{S}=$ non-autoclaved supernatant fluid). A whole set of reflectance spectra are provided in Fig. S1 in the ESI. $\dagger$

the photographs. The reflectance intensity at blue $(640 \mathrm{~nm})$ and red $(540 \mathrm{~nm})$ on the spectra was used in calculating colorimetric response percentages (CR\%) for the nanofibers tested with different $E$. coli culture components. Fig. 6c shows the $\mathrm{CR} \%$ as a function of testing time for the nanofibers tested. To see a significant color transition in the nanofiber, it took $3 \mathrm{~min}$ which confirms the sensitivity of the system discussed previously. The colorimetric transition was visible at $t=3 \mathrm{~min}$, continued increasing, and plateaued, resulting in a peak colorimetric transition of $28 \%$ for the autoclaved supernatant fluid and $10 \%$ for the non-autoclaved supernatant fluid, respectively. The CR\% was negligible for the PU-PDA nanofiber mats tested with $E$. coli cell pellets from $t=0$ to $t=24 \mathrm{~h}$.

\section{Colorimetric response of nanofibers to supernatant fluid with increased storage time}

Supernatant fluids with different storage times $\left(t_{\mathrm{s}}=0,24\right.$, and $36 \mathrm{~h}$ ) were evaluated for the ability to activate a colorimetric transition in the PU-PDA nanofiber. Components in the supernatant fluid such as carbohydrates and proteins can undergo structural changes such as degradation over time as no new metabolites are produced in the absence of bacterial cells. Table 2 shows the photographs of PU-PDA nanofiber mats before and after testing with supernatant fluid with different storage times $\left(t_{\mathrm{s}}\right)$. Supernatant fluid with increased storage time changed the color of nanofibers significantly slower than the supernatant fluid with no storage time, suggesting degradation in CCS with increased storage. In addition, the degradation of CCS in autoclaved supernatant fluid with $t_{\mathrm{s}}=36 \mathrm{~h}$ was significant because the vibrant red color was absent in the nanofibers, which was not observed in non-autoclaved supernatant fluid with $t_{\mathrm{s}}=36 \mathrm{~h}$ (Table 2).

Fig. 6d shows CR\% of the nanofibers tested with supernatant fluid with different storage times $\left(t_{\mathrm{s}}\right)$. Generally, color change started at $t=3 \mathrm{~min}$ and then increased in CR\% followed by a plateau at $t=180 \mathrm{~min}$. A reduction in CR\% was found with an increase in the storage time of supernatant fluid, which

Table 2 Photographs of PU-PDA nanofibers before and after testing with supernatant fluid with different storage times

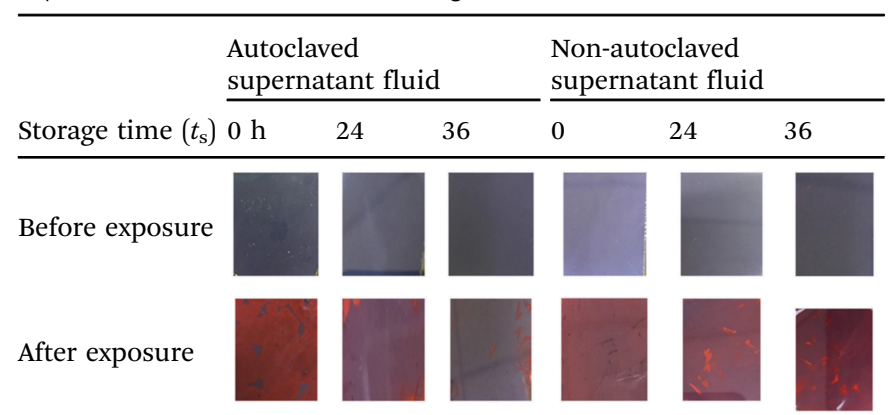


agrees with the photographs presented in Table 2 . The CR\% at the plateau in the case of autoclaved supernatant fluid was $28 \%$ at $t_{\mathrm{s}}=0 \mathrm{~h}, 14 \%$ at $t_{\mathrm{s}}=24 \mathrm{~h}$, and $2.5 \%$ at $t_{\mathrm{s}}=36 \mathrm{~h}$, respectively. The CR\% at the plateau decreased by almost $25.5 \%$ from $t_{\mathrm{s}}=0 \mathrm{~h}$ to $t_{\mathrm{s}}=36 \mathrm{~h}$. On the other hand, the non-autoclaved supernatant fluid demonstrated CR\% at the plateau of $10 \%$ at $t_{\mathrm{s}}=0 \mathrm{~h}, 5 \%$ at $t_{\mathrm{s}}=24 \mathrm{~h}$, and $3 \%$ at $t_{\mathrm{s}}=36 \mathrm{~h}$, respectively. $\mathrm{CR} \%$ at the plateau decreased by almost $7 \%$.

\section{Colorimetric response of nanofibers to size-fractionated supernatant fluid}

The supernatant fluid is a complex mixture of monomers and biopolymers having different molecular weights. To determine a specific molecular weight range, the $E$. coli supernatant fluid was size-fractionated and tested with PU-PDA nanofibers. Table 3 shows the photographs of PU-PDA nanofibers tested with size-fractionated supernatant fluid. The color change was found for all fluids that passed through a filter with a molecular weight cutoff of $3 \mathrm{k}, 30 \mathrm{k}$, and $100 \mathrm{k}$ Da. The color change was also observed for the fluid that could not pass through the filter having a molecular weight cutoff of $100 \mathrm{k} \mathrm{Da}$. This result suggested that the CCS was composed of multimetric substances that were not a single component in the fluid, but multiple components with different molecular weights.

\section{Colorimetric response of nanofibers to extracellular polymeric substances (EPS)}

The bacterial supernatant fluid is essentially made of materials produced by bacteria that are collectively known as extracellular polymeric substances (EPS). The EPS produced by E. coli can broadly be classified as bound and free EPS. The bound EPS remains attached to the $E$. coli cell and the free EPS independently floats in the biofilm. ${ }^{21}$ As discussed previously, E. coli cell pellets did not induce any color change in PU-PDA nanofibers, which indicates that the CCS was not the bound EPS. Therefore, free EPS were extracted from both autoclaved and non-autoclaved supernatant fluid to test with nanofibers. Table 4 shows the color change behaviors of the nanofibers immersed in free EPS solutions. The free EPS extracted from both autoclaved and non-autoclaved supernatant fluid changed the color of nanofibers. Due to the superhydrophobic nature of the PU-PDA nanofiber mat, the free EPS solution could not
Table 4 Photographs of PU-PDA nanofibers before and after testing with E. coli culture components and free EPS. $S=$ non-autoclaved supernatant fluid, $A S=$ autoclaved supernatant fluid, $P=$ non-autoclaved bacterial cell pellet, $\mathrm{AP}=$ autoclaved bacterial cell pellet, $\mathrm{LB}=\mathrm{LB}$ medium solution (control)

\begin{tabular}{|c|c|c|}
\hline \multirow[b]{2}{*}{ E. coli component } & \multicolumn{2}{|c|}{ Test with free EPS } \\
\hline & AS & $\mathrm{S}$ \\
\hline Before exposure & & \\
\hline After exposure & & \\
\hline
\end{tabular}

penetrate the nanofiber fiber mat completely. In addition, the concentration of CCS in free EPS might not be as high as in the supernatant fluid. For these reasons, some portion of the nanofiber remained blue after free EPS exposure as shown in the photographs. The difference in the red color between the nanofibers treated with free EPS collected from autoclaved and non-autoclaved supernatant fluids can be a result of structural differences in CCS due to the autoclaving process. The response time of color changes was $30 \mathrm{~min}$ in the free EPS extracted from autoclaved supernatant fluid and $3 \mathrm{~h}$ for the non-autoclaved fluid, respectively. To the best of our knowledge, it is the first report of testing PDAs with free EPS extracted from bacterial culture, which enhanced the understanding of the response mechanism in bacteria-sensing PDA biosensors.

\section{Colorimetric response of nanofibers to $E$. coli culture components from M9 minimal media}

There are different secretion pathways for a Gram-negative bacterium such as $E$. coli and these secretion pathways can change with variations in the surrounding environment such as culture media. ${ }^{36}$ M9 minimal medium contains only salts and a nitrogen source and is often used to study the nutritional aspects of $E$. coli growth when supplemented with different carbon sources and amino acids. M9 minimal medium is a defined growth medium that lacks the peptides, amino acids, vitamins, and trace elements found in LB media. In this experiment, M9 minimal medium was supplemented with arabinose and thiamine (due to

Table 3 Photographs of PU-PDA nanofibers before and after testing with supernatant and autoclaved supernatant fluids not retained by filters with molecular weight cutoffs of 3k, 30k, and 100k Da, and fluid that was retained by a filter with a molecular weight cutoff of $100 \mathrm{k}$ Da

\begin{tabular}{|c|c|c|c|c|c|c|c|c|}
\hline & Molec & cutoffs & & & & & & \\
\hline & $<3 \mathrm{k}$ & $<30 \mathrm{k}$ & $<100 \mathrm{k}$ & $>100 \mathrm{k}$ & $<3 \mathrm{k}$ & $<30 \mathrm{k}$ & $<100 \mathrm{k}$ & $>100 \mathrm{k}$ \\
\hline Before & & & & & & & & \\
\hline After & & & & & & & & \\
\hline
\end{tabular}


Table 5 Photographs of PU-PDA nanofibers before and after testing with E. coli culture components from M9 minimal media. $S=$ non-autoclaved supernatant fluid, $A S=$ autoclaved supernatant fluid, $P=$ non-autoclaved bacterial cell pellet, $A P=$ autoclaved bacterial cell pellet, $M 9=M 9$ minimal medium solution (control)

\begin{tabular}{llllll}
\hline Bacterial components & Control: M9 & AS & S & AP & P \\
\hline Before exposure & & & & & \\
& & & & & \\
After exposure & & & & &
\end{tabular}

the auxotrophy of $E$. coli) and different $E$. coli culture components were tested with the PU-PDA nanofibers. The photographs from the result are shown in Table 5. No color changes were promoted by any of the culture components from M9 minimal media. The result is significantly different from the experiment with LB media and most likely, the minimal nutritional components that $E$. coli received from M9 minimal media were not enough to produce the CCS responsible for a color change in the PU-PDA nanofibers.

\section{Conclusions}

A simple method of electrospinning was reported to fabricate molecular receptor-free PDA nanofiber biosensors that demonstrated a rapid blue-to-red change in detecting $E$. coli with a response time of $0.5 \mathrm{~min}$ to $3 \mathrm{~min}$ and a critical bacterial concentration $(\mathrm{CBC})$ of $9 \times 10^{8} \mathrm{CFU} \mathrm{mL}^{-1}$. The receptor-free PDA nanofiber biosensors attributed high sensitivity of bacterial detection via colorimetric transition to a highly ordered and self-assembled PDA molecular vesicle structure that was promoted and enhanced in the electrospinning process due to large electrostatic forces. The PDA nanofiber composites also showed solvatochromism in response to different organic solvents with different colorimetric response percentage, suggesting a potential sensor application in fingerprinting organic solvents. The nature of the color-changing substances (CCS) in the complex $E$. coli culture was investigated. The colorimetric response of the nanofibers was found with the supernatant fluid and free EPS. The CCS that was produced in rich culture media such as LB media was unlikely a protein, DNA, RNA, or LPS and was likely the composition of multimetric components with different molecular weights. Interestingly, when $E$. coli received minimum nutrition, it changed the secretion system and thus altered the colorimetric transition behavior of the PDA nanofiber system. The CCS was not present in E. coli grown in a minimum nutrition medium (M9 minimal medium) because no color change was found in the PDA. On the other hand, the color-changing rate of the nanofibers decreased significantly with increasing storage time, suggesting that the CCS degraded or lost the ability to interact with the PU-PDA nanofibers with an increase of storage time.

\section{Conflicts of interest}

The authors declare no potential conflict of interest concerning the research, authorship, and/or publication of this article.

\section{Acknowledgements}

This work was supported by the funding from the Colorado State University College Research Council and the AATCC Graduate Student Research Support Grant provided by the American Association of Textile Chemists and Colorists (AATCC).

\section{References}

1 S. Günes, H. Neugebaur and N. Sariciftci, Chem. Rev., 2007, 107, 1324-1338.

2 Q. Pei, G. Yu, C. Zhang, Y. Yang and A. Heeger, Science, 1995, 269, 1086-1088.

3 K. M. Coakley and M. D. McGehee, Chem. Mater., 2004, 16, 4533-4542.

4 X. Feng, L. Liu, S. Wang and D. Zhu, Chem. Soc. Rev., 2010, 39, 2411-2419.

5 E. Smela, Adv. Mater., 2003, 15, 481-494.

6 C. Kallinger, M. Hilmer, A. Haugeneder, M. Perner, W. Spirkl, U. Lemmer, J. Feldmann, U. Scherf, K. Müllen, A. Gombert and V. Wittwer, Adv. Mater., 1999, 10, 920-923.

7 D. T. McQuade, A. E. Pullen and T. M. Swager, Chem. Rev., 2000, 100, 2537-2574.

8 X. Qian and B. Städler, Chem. Mater., 2019, 31, 1196-1222.

9 D. H. Charych, J. O. Nagy, W. Spevak and M. D. Bednarski, Science, 1993, 261, 585-588.

10 L. Li, X. An and X. Yan, Colloids Surf., B, 2015, 134, 235-239.

11 M. Ulaganathan, R. V. Hansen, N. Drayton, H. Hingorani, R. G. Kutty, H. Joshi, S. Sreejith, Z. Liu, J. Yang and Y. Zhao, ACS Appl. Mater. Interfaces, 2016, 8, 32643-32648.

12 L. Chen, F. Wu, Y. Li, Y. Wang, L. Si, K. I. Lee and B. Fei, J. Membr. Sci., 2018, 547, 93-98.

13 A. K. M. M. Alam, J. P. Yapor, M. M. Reynolds and Y. V. Li, Materials, 2016, 9, 202.

14 J. P. Yapor, A. Alharby, C. Gentry-Weeks, M. M. Reynolds, A. K. M. M. Alam and Y. V. Li, ACS Omega, 2017, 2, 7334-7342.

15 A. St John and C. P. Price, Clin Biochem Rev, 2014, 35, 155-167.

16 S. U. Son, S. B. Seo, S. Jang, J. Choi, J. Lim, D. K. Lee, H. Kim, S. Seo, T. Kang, J. Jung and E.-K. Lim, Sens. Actuators, B, 2019, 291, 257-265.

17 C. H. Park, J. P. Kim, S. W. Lee, N. L. Jeon, P. J. Yoo and S. J. Sim, Adv. Funct. Mater., 2009, 19, 3703-3710.

18 P. Villalobos, M. I. Chavez, Y. Olguín, E. Sánchez, E. Valdés, R. Galindo and M. E. Young, Electron. J. Biotechnol., 2012, 15, 4-15.

19 K.-W. Kim, H. Choi, G. S. Lee, D. J. Ahn and M.-K. Oh, Colloids Surf., B, 2008, 66, 213-217.

20 A. C. dos Santos Pires, N. de Fatima Ferreira Soares, L. H. M. da Silva, M. do C. H. da Silva, M. V. De Almeida, 
M. Le Hyaric, N. J. de Andrade, R. F. Soares, A. B. Mageste and S. G. Reis, Sens. Actuators, B, 2011, 153, 17-23.

21 K. E. Eboigbodin and C. A. Biggs, Biomacromolecules, 2008, 9, 686-695.

22 X. Zhang, Fundamentals of Fiber Science, DEStech Publications Inc., Lancaster, PA, 2014.

23 N. Bhardwaj and S. C. Kundu, Biotechnol. Adv., 2010, 28, 325-347.

24 F. Ko, Y. Gogotsi, A. Ali, N. Naguib, H. Ye, G. L. Yang, C. Li and P. Willis, Adv. Mater., 2003, 15, 1161-1165.

25 C. F. Holder and R. E. Schaak, ACS Nano, 2019, 13, 7359-7365.

26 S. K. Chae, H. Park, J. Yoon, C. H. Lee, D. J. Ahn and J.-M. Kim, Adv. Mater., 2007, 19, 521-524.

27 F. Hassan, C. Gentry-Weeks, M. M. Reynolds and Y. V. Li, J. Appl. Polym. Sci., 2019, 136, 47877-47890.

28 M. F. Freshwater and C. T. Su, Ann. Plast. Surg., 1980, 4, 216-218.

29 M. C. Robson, R. J. Mannari, P. D. Smith and W. G. Payne, Am. J. Surg., 1999, 178, 399-402.
30 S. Pumtang, W. Siripornnoppakhun, M. Sukwattanasinitt and A. Ajavakom, J. Colloid Interface Sci., 2011, 364, 366-372.

31 H. Jiang, Y. Wang, Q. Ye, G. Zou, W. Su and Q. Zhang, Sens. Actuators, B, 2010, 143, 789-794.

32 W. Wu, J. Zhang, M. Zheng, Y. Zhong, J. Yang, Y. Zhao, W. Wu, W. Ye, J. Wen, Q. Wang and J. Lu, PLoS One, 2012, 7, e48999, DOI: 10.1371/journal.pone.0048999.

33 T. V. de Oliveira, N. de F. F. Soares, D. J. Silva, N. J. de Andrade, E. A. A. Medeiros and A. T. Badaró, Sens. Actuators, $B, 2013$, 188, 385-392.

34 H. Neurath, J. P. Greenstein, F. W. Putnam and J. A. Erickson, Chem. Rev., 1944, 34, 157-265.

35 M. A. Valvano, O Antigen Biosynthesis, in Comprehensive Natural Products II, ed. H.-W. B. Liu and L. Mander, Elsevier, USA, 2010, pp. 297-314, DOI: 10.1016/B978-0080453828.00683-3.

36 A. W. Maresso, Bacterial Secretion Systems, in Bacterial Virulence-A Conceptual Primer, Springer Nature Switzerland AG, Cham, Switzerland, 2019, pp. 103-114, DOI: 10.1007/ 978-3-030-20464-8_9. 